\title{
INNOVACIÓN EN EL PERIODISMO EMPRENDEDOR DEPORTIVO. MODELO DE NEGOCIO Y NARRATIVAS
}

\author{
New media innovation: the case of entrepreneurial \\ sports journalism
}

\author{
Juan-Luis Manfredi-Sánchez, José-Luis Rojas-Torrijos y José-María Herranz- \\ de-la-Casa
}

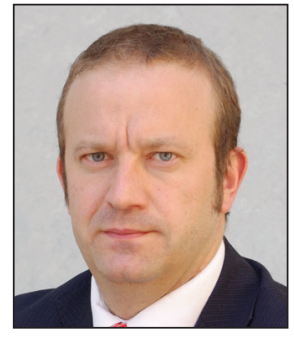

Juan-Luis Manfredi-Sánchez es profesor contratado doctor de periodismo de la Universidad de Castilla-La Mancha. Ha dirigido Periodismo emprendedor, una esperanza renovada, contrato de I+D de la Universidad de Castilla-La Mancha con financiación del Departamento de Estado de Estados Unidos. Es el investigador principal del proyecto Comunicación pública, transparencia, rendición de cuentas y participación en los gobiernos locales, GlobalCOM (CSO2013-46997-R). http://orcid.org/0000-0001-9129-2907

Universidad de Castilla-La Mancha, Facultad de Periodismo Campus universitario, s/n. 16071 Cuenca, España juan.manfredi@uclm.es

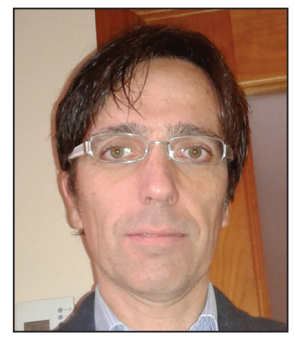

José-Luis Rojas-Torrijos es profesor asociado de periodismo de la Universidad de Sevilla y profesor de periodismo en el centro universitario EUSA. Compagina su labor docente e investigadora con la de periodista en la Oficina del Portavoz del Gobierno de la Junta de Andalucía. Forma parte del grupo de investigación Estudio de medios para un periodismo de calidad.

http://periodismodeportivodecalidad.blogspot.com

http://orcid.org/0000-0002-7390-9843

Universidad de Sevilla, Facultad de Comunicación Avda. Américo Vespucio, s/n. Isla de la Cartuja. 41092 Sevilla, España jlrojas@us.es

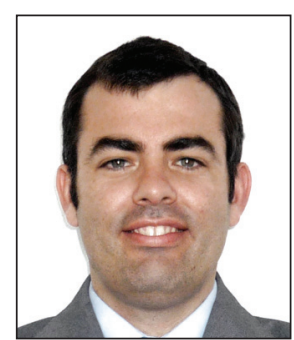

\section{Resumen}

El periodismo emprendedor es el conjunto de iniciativas creadas desde 2008 por periodistas para afrontar la transformación del mercado periodístico español. La especialización en el deporte se ha convertido en uno de los ejes de crecimiento, copando alrededor del $17 \%$ del mercado de nuevos medios. Se evalúa el grado de innovación producido en la producción periodística. La metodología utiliza dos variables: la propuesta de valor, en particular el modelo de negocio, y la narrativa periodística. Se han censado 65 nuevos medios y se han cruzado ambas variables. Se observa que los nuevos proyectos que emplean géneros tradicionales (noticias, servicios informativos) son menos innovadores en la búsqueda de modelos de negocio. A la inversa, la apuesta por géneros diferentes (lectura pausada) va de la mano de modelos no basados sólo en la publicidad y la venta de ejemplares. Se concluye que hay poca innovación en 41 proyectos de noticias deportivas, y se destacan al menos 8 iniciativas con una propuesta innovadora.

José-María Herranz-de-la-Casa es doctor en periodismo por la Universidad Complutense de Madrid. Ha trabajado como periodista en el diario deportivo Marca y ha sido profesor de la Universidad Católica de Ávila (UCAV) y de la Universidad Europea Miguel de Cervantes de Valladolid. En ambas universidades ha sido también director del Gabinete de Comunicación y Marketing. Es profesor de la Universidad de Castilla-La Mancha y director de la plataforma digital El observador de Castilla-La Mancha de la Facultad de Periodismo.

http://observador.uclm.es

http://orcid.org/0000-0002-3667-2664

Universidad de Castilla-La Mancha, Facultad de Periodismo Campus universitario, s/n. 16071 Cuenca, España josemaria.herranz@uclm.es 


\title{
Palabras clave
}

Nuevos medios; Periodismo emprendedor; Periodismo deportivo; Periodismo digital; Nuevas narrativas; Innovación.

\begin{abstract}
Entrepreneurial journalism is the set of projects created since 2008 by journalists to deal with the transformation of the Spanish media market. Specialization in sports stands out as one of the levers of growth, constituting around 17 per cent of the new media outlets. The degree of innovation in journalism production are analyzed through the measurement of two elements: their value proposition, in particular their business model, and the new ways of storytelling deployed in journalism. We identified and assessed 65 new media according to their degree of innovation. We concluded that new projects generating traditional genres (news, information services) are less innovative in the pursuit of business models. Conversely, the use of different genres (slow journalism) goes hand in hand with models not based only on advertising and sales. In conclusion, while there is little innovation in 41 projects of sports news, at least 8 initiatives with an innovative proposal stand out.
\end{abstract}

\section{Keywords}

New media; Entrepreneurial journalism; Sports journalism; Digital journalism; Digital storytelling; Innovation

Manfredi-Sánchez, Juan-Luis; Rojas-Torrijos, José-Luis; Herranz-de-la-Casa, José-María (2015). “Innovación en el periodismo emprendedor deportivo. Modelo de negocio y narrativas". El profesional de la información, v. 24, n. 3, pp. 265-273.

http://dx.doi.org/10.3145/epi.2015.may.06

\section{Introducción}

Se plantean tres objetivos de investigación.

El primero consiste en el análisis de la consolidación de los estudios en materia de periodismo deportivo en España (Manfredi-Sánchez; Rojas-Torrijos; Herranz-de-la-Casa, 2015; Rojas-Torrijos, 2011; 2013; Barrero-Muñoz, 2008; Alcoba-López, 2005). A pesar de la popularidad de los deportes y su presencia masiva en los medios convencionales y en los digitales, no existe un corpus investigador sólido en esta área de especialización periodística. Los estudios universitarios sobre asuntos deportivos se centran en otras áreas (gestión, fisiología, economía). Destaca el Centro de Estudios Olímpicos, ubicado en la Universidad Autónoma de Barcelona, y la Cátedra Real Madrid, en la Universidad Europea de Madrid. Ninguna de las dos cuenta con una trayectoria extensa en temas periodísticos.

El segundo objetivo es el análisis del fenómeno del periodismo emprendedor en España. Consiste en el conjunto de iniciativas lideradas por periodistas para la creación de nuevos medios. Se caracterizan por la primacía del modo digital en detrimento de la estructura tradicional. El periodismo deportivo es uno de los ejes de crecimiento de los nuevos medios: desde 2008, se han creado 65 proyectos periodísticos, alrededor del $17 \%$ del censo de nuevos medios elaborado por la Asociación de la Prensa de Madrid (2014). Este trabajo continúa la línea investigadora de Manfredi-Sánchez (2015), Goyanes; Peinado (2014), Paniagua-Rojano; Gómez-Aguilar; González-Cortés (2014), Casero-Ripollés; Cullell-March (2013) y Cabezuelo-Lorenzo (2013).

El tercer objetivo es el estudio de la innovación periodística en los nuevos medios. En la empresa, se dan tres tipos:

- creación de nuevos mercados

- sostenibilidad o mejora incremental

- eficiencia de los procesos o en la gestión.
En el periodismo, la innovación se clasifica en las áreas de: -producto y servicio periodístico; -producción, comercialización y distribución; -organización.

La innovación presenta oportunidades de transformación del negocio, pero también barreras (Chesbrough, 2010).

Estos tres objetivos de investigación conducen al planteamiento de la siguiente hipótesis: existe una relación directa entre el grado de innovación periodística y el tipo de género periodístico empleado. Así, los nuevos medios que son innovadores en la narrativa periodística (géneros, empleo intensivo de datos, infografía, análisis y prospectiva, lectura pausada) también han innovado en la propuesta de valor (diseño, ilustración y géneros) y el modelo de negocio (ingresos). En cambio, los nuevos proyectos que emplean géneros tradicionales (noticias, crónica, actualidad) se sustentan sobre modelos de negocio propios del entorno tradicional (publicidad, suscripciones).

\section{Metodología}

\subsection{Marco teórico}

La metodología empleada consiste en la identificación de los nuevos medios creados en España desde 2008, bajo el paraguas del fenómeno denominado periodismo emprendedor. Se identifican cinco elementos que definen estos nuevos proyectos:

- propiedad y titularidad del nuevo medio en manos de periodistas;

- entorno digital y móvil;

- marca personal de los profesionales;

- naturaleza de la propuesta de valor;

- uso de nuevas narrativas periodísticas.

La naturaleza innovadora de los nuevos medios se basa en su naturaleza digital y la orientación de la inversión hacia la 
producción periodística (Manfredi-Sánchez; Artero, 2014, p. 161). Esta investigación se concentra en el cuarto y el quinto elementos.

\section{Propuesta de valor}

Consiste en la conceptualización, diseño y ejecución del proyecto periodístico; en la descripción del producto informativo, el modelo de negocio, la relación con los lectores, los anunciantes y los mercados publicitarios, la elección de una tecnología, la comercialización, y la organización de la redacción, entre otros. La propuesta de valor es el eje de la estrategia en la creación de proyectos empresariales, porque compendia la identidad, la misión y el producto periodístico. Exige el análisis y la medición de elementos objetivos, públicos y concretos. Por eso, el modelo de negocio (Hedman; Kalling, 2003) sintetiza la propuesta de valor y permite identificar patrones de relación con los lectores, los anunciantes y los propios periodistas.

El desarrollo del modelo de negocio establece un sistema de ingresos, que es la respuesta a quién paga por los servicios y productos que se ofrecen en el mercado. No existen modelos puros, sino que suelen ser el resultado de la combinación de varios sistemas. En la prensa convencional, de inspiración fordista ${ }^{1}$, el modelo de negocio se basa en el control sobre la distribución a través de una red capilar de puntos de venta, las suscripciones y la captación de inversiones publicitarias. La audiencia masiva genera más ingresos que el periodismo de nicho en términos absolutos, aunque tal relación no mida la rentabilidad de los proyectos. El modelo de negocio es un elemento objetivo, que se puede medir y evaluar sin la intervención de los interesados.

\section{Los proyectos emprendedores en perio- dismo deportivo no han seguido una lí- nea innovadora}

Sin embargo, en la prensa digital los ingresos proceden de diversas fuentes. No existe un patrón unificado de negocio como el señalado para la prensa de papel. La evolución ha sido lenta en los medios convencionales que crean estrategias para internet y los nuevos medios. Casero-Ripollés en un estudio temprano (2010) analiza cómo dos medios convencionales (El país y El mundo) y dos medios nativos (Soitu y Factual) emplean la misma estrategia. En este contexto, parece oportuno explorar nuevas vías de financiación. Yuste y Cabrera (2014, pp. 87-132) enumeran en su estudio la venta de contenidos por internet, la publicidad, el patrocinio, el crowdfunding, micropagos, suscripciones, cupones y descuentos, apps y tabletas, versioning, sindicación de contenidos, servicio de selección de contenidos o generación de contenidos a medida.

La innovación en el modelo de negocio es posterior y, probablemente, catalizado por el declive del mercado publicitario (Infoadex, 2014). La arquitectura del emprendimiento periodístico se extiende hacia componentes propios del negocio digital, que adapta la propuesta de valor y transforma las capacidades de la compañía. Interesa aquí el reciente ranking de innovación periodística elaborado por la Universidad Miguel Hernández de Elche, que persigue la creación de una metodología de medición de la innovación (UMH, 2014).

\section{Narrativa periodística}

Consiste en la seña de identidad del periodismo que dota de sentido a la profesión. La diferenciación reside en cómo contar las historias, en explicar los detalles de lo sucedido, y en cómo presentar la información, de forma que ésta resulte lo más atractiva y original para alcanzar al público al que se dirige. La innovación en la narrativa transforma el producto y el servicio periodístico que se ofrece a los lectores: encontramos una evolución permanente en los géneros y formatos periodísticos más tradicionales, que han pasado a replantearse la forma y el contenido, y de esta manera poder aprovechar las nuevas posibilidades narrativas que ofrece el entorno digital y adaptarse mejor a los nuevos hábitos de consumo de los usuarios, cada vez más presentes en los dispositivos móviles.

\subsection{Diseño de la investigación}

En primer lugar, se han censado los medios deportivos españoles de nueva creación. Esta clasificación informativa suele ser especializada de acuerdo con el ámbito de difusión que rige. Así, encontramos diarios dedicados principalmente a un deporte (fútbol), un equipo (Sevilla FC o Real Betis) o una categoría (fútbol profesional, deporte municipal). Tal especialización refuerza la idea de nicho de audiencia y facilita la multiplicación de iniciativas periodísticas que juegan con dos criterios relevantes: geográficos y temáticos.

El censo se compone de 65 nuevos medios, que han sido lanzados desde 2008 hasta finales de 2014. Se han mantenido en el listado todos, aunque algunos cerraron o están inaccesibles (tabla 1).

A continuación se han establecido tres criterios de innovación.

a) Producto o servicio periodístico: ante un mercado informativo saturado, se apuesta por otros géneros menos vinculados a la actualidad noticiosa y se exploran nuevas narrativas y formatos.

Entre los géneros que facilitan la innovación encontramos el reportaje en profundidad, el análisis, los informes y la entrevista extensa no vinculada a los resultados de la jornada de competición regular. Prima el género interpretativo que mezcla el hecho informativo con la opinión, la visión del redactor;

b) Uso de técnicas como la infografía, diseño o bases de datos. Es poco habitual en medios convencionales o bien en medios de naturaleza digital que siguen el patrón de aquellos.

c) Propuesta de valor, con especial atención al modelo de negocio. La captación de publicidad se considera poco innovadora. En cambio, la apuesta por la comunidad de lectores o los productos premium en tienda sí lo son. Otras fórmulas de financiación innovadoras son el crowdfunding, elaboración de aplicaciones para tabletas y teléfonos, alianzas, par- 
Tabla 1. Censo de nuevos medios deportivos (2008-2014)

\begin{tabular}{|c|c|c|}
\hline Medio & Web & Año de creación \\
\hline Viva futbol & http://www.vivafutbol.es & 2010 \\
\hline Ecos del balón & http://www.ecosdelbalon.com & 2011 \\
\hline El desmarque & http://www.eldesmarque.com & 2006 \\
\hline Sportyou & http://www.sportyou.es & 2008 \\
\hline Sports made in USA & http://sportsmadeinusa.com & 2009 \\
\hline Vavel & http://www.vavel.com/es & 2010 \\
\hline Once contra once & http://www.actiweb.es/oncecontraonce & 2010 \\
\hline Piratas del basket & http://piratasdelbasket.net & 2010 \\
\hline Vivafutbol & http://www.vivafutbol.es & 2010 \\
\hline Sportics & http://sportics.es & $\begin{array}{c}2011 \\
\text { Paralizado desde junio } 2014\end{array}$ \\
\hline DeporPress & http://www.deporpress.com & 2011 \\
\hline Master1000 & http://master1000.es & 2011 \\
\hline Sentimiento AyN & http://www.sentimientoayn.com & 2011 \\
\hline Panenka & http://www.panenka.org & 2011 \\
\hline Ecos del balon & http://www.ecosdelbalon.com & 2011 \\
\hline Munideporte & http://www.munideporte.com & 2003 \\
\hline Quality sport & & $\begin{array}{c}2011 \\
\begin{array}{c}\text { Cerró en 2014. Su equipo pasó a formar parte de Marca } \\
\text { plus }\end{array}\end{array}$ \\
\hline Tú al Ramiro y yo a Badalona & http://www.ramirobadalona.com & $\begin{array}{c}2011 ? \\
\text { Cerró en diciembre de } 2013\end{array}$ \\
\hline Camp d'esports & http://www.campdesports.cat & 2012 \\
\hline Canarias basket & http://canariasbasket.com & 2012 \\
\hline Cronómetro deportivo & http://www.cronometrodeportivo.es & 2012 \\
\hline Cuadernos del basket & http://www.cuadernosdebasket.com & 2012 \\
\hline Deporadictos & http://deporadictos.com & 2012 \\
\hline Radio goles & http://www.radiogoles.es & 2012 \\
\hline Kaiser football magazine & http://www.kaisermagazine.com & 2012 \\
\hline Pasaporte olímpico & http://issuu.com/podium 1896/docs/revista_p_dium_num.01 & $\begin{array}{c}2012 \\
\text { Edita revista Pódium (2014) }\end{array}$ \\
\hline El deporte conquense & http://eldeporteconquense.com & 2012 \\
\hline Protagonistas del juego & http://protagonistasdeljuego.com & 2012 \\
\hline Eleven foot & & $\begin{array}{c}2012 \\
\text { Cerró en agosto de } 2013 \\
\end{array}$ \\
\hline Diario gol & http://www.diariogol.com & 2012 \\
\hline HockeyDH & http://www.hockeydh.com & 2012 \\
\hline Lineker magazine & http://www.linekermagazine.es & 2012 \\
\hline Spanish player go & http://www.spanishplayer.com & 2012 \\
\hline Falso 9 & http://falso9.com & 2012 \\
\hline Ha10 & http://ha10.es & 2012 \\
\hline Juegos Rio Janeiro 2016 & http://www.juegosriojaneiro2016.com & 2012 \\
\hline Libero & http://shop.revistalibero.com & 2012 \\
\hline Marti Perarnau & http://www.martiperarnau.com & 2012 \\
\hline Mundial de futbol 2014 & http://www.mundialdefutbol2014.es & 2012 \\
\hline Mas que alba & http://www.masquealba.com & 2013 \\
\hline Palabra de futbol & http://palabradefutbol.com & 2013 \\
\hline Proyecto premier & http://www.proyectopremier.com & 2013 \\
\hline Ritmo de juego & Desaparecido & 2013 \\
\hline Sphera sports & http://www.spherasports.com & 2013 \\
\hline Alirón & http://www.alironbilbao.com & 2013 \\
\hline La tribuna deportiva & http://latribunadeportiva.net & 2013 \\
\hline Deporte Sevilla & http://deportesevilla.tv & 2013 \\
\hline Gipuzkoa sport & http://www.gipuzkoasport.com & 2013 \\
\hline El juego de Naismith & http://www.eljuegodenaismith.com & 2013 \\
\hline
\end{tabular}


Tabla 1 (cont.). Censo de nuevos medios deportivos (2008-2014)

\begin{tabular}{|c|c|c|}
\hline Medio & Web & Año de creación \\
\hline Time out magazine & http://www.timeout.com & 2013 \\
\hline La jugada financiera & http://lajugadafinanciera.com & 2013 \\
\hline Minuto 116 & http://revistaminuto116.com & 2013 \\
\hline Metadeporte & http://metadeporte.com & 2013 \\
\hline Maratón radio & http://www.maratonradio.com & 2013 \\
\hline Avance deportivo & http://www.avancedeportivo.es & 2013 \\
\hline Ciclo 21 & http://www.ciclo21.com & 2013 \\
\hline Los otros 18 & http://www.losotros18.com & 2013 \\
\hline Diario de La roja & Desaparecido & 2014 \\
\hline Mundo sala & http://futsalia.es & $\begin{array}{l}2014 \\
\text { Cerró en junio de } 2014 \text {, y renació como Futsalia }\end{array}$ \\
\hline Deporte para todos & http://www.deporteparatodos.net & 2014 \\
\hline KIAenzona & http://kiaenzona.com & 2014 \\
\hline La Fosbury & http://fosbury.cat/\#!menu & 2014 \\
\hline Olympo Deportivo & http://www.olympodeportivo.es & 2014 \\
\hline Volata & http://volatamag.cc & 2014 \\
\hline El enganche & http://www.elenganche.es & 2014 \\
\hline Revista Elite sport & http://www.revistaelitesport.es & 2014 \\
\hline Estadio Leiva & http://estadioleiva.com & 2014 \\
\hline
\end{tabular}

tenariado con medios convencionales, filantropía o la sindicación de contenidos, entre otras.

Por último, se han cruzado los datos para valorar la hipótesis inicial. El conteo es manual en la medida que el equipo investigador ha revisado los 65 medios censados y ha completado la información de los siguientes items:

- nombre y web de la publicación;

- especificidad geográfica;

- especificidad deportiva;

- género de portada (visita diaria durante una semana para conocer el grado de innovación en el producto no noticioso);

- innovación en el negocio (es gradual; desde la ausencia de innovación que es la publicidad y la venta de ejemplares, hasta la creación de comunidades);

- innovación en el mercado periodístico (creación de un producto o servicio para un segmento no atendido).

Se ha concluido con una valoración del grado de innovación (incremental o sustancial) en las áreas de producto y servicio periodístico; producción, comercialización y distribución; y organización.

El período de investigación ha abarcado de diciembre de 2014 a enero de 2015, dos meses de actividad informativa que incluye liga regular, parón invernal o actividades extraordinarias. Es una muestra significativa de la actividad anual.

\section{Resultados}

Los hallazgos confirman la hipótesis planteada en la pregunta de investigación. De forma concluyente, se observa que los proyectos emprendedores en periodismo deportivo no han seguido una línea innovadora. Al contrario, han transpuesto modelos periodísticos conservadores (noticias y servicios informativos) que heredan una propuesta de valor de corte tradicional. Esto explica que 41 de los proyectos listados apuesten por las noticias y la actualidad como género de portada, es decir, como elemento principal de la web. Este hecho redunda en un diseño estándar, poco diferenciado de otros medios convencionales del ámbito deportivo. También significa: enumeración de noticias, columna lateral con opinión destacada y conexión con redes sociales. Sorprende el escaso grado de especialización y diversificación de los proyectos: 33 se orientan hacia el fútbol profesional, 11 a temas polideportivos y 7 a baloncesto. Los otros 14 se reparten entre polideportivo, hockey y otros deportes minoritarios. No se ha encontrado en el censo actividad relacionada con los deportes de motor o nieve, cuya popularidad se estima sobresaliente.

En relación con el modelo de negocio, 45 nuevos medios han optado por la publicidad, un sistema convencional y poco innovador en el entorno digital. Destaca la elección de fórmulas poco avanzadas, como la publicidad invasiva, los banners o los robapáginas. En este punto, se han encontrado fórmulas mixtas de financiación. Casi todos los medios reseñados plantean varias vías de ingresos. Como indicador de innovación interesa más la propuesta de valor, esto es, la identificación de un proyecto periodístico diferente antes que la inclusión de publicidad.

a) Género de portada (sobre 65 censados)

- 41 noticias

- 6 contenidos largos (long-form)

- 3 opinión e interpretación

- 3 radio

- 2 vídeo

- 2 banco de datos, informes

- 1 vintage

- 1 red de blogs

- 1 reportaje

- 5 no activo 


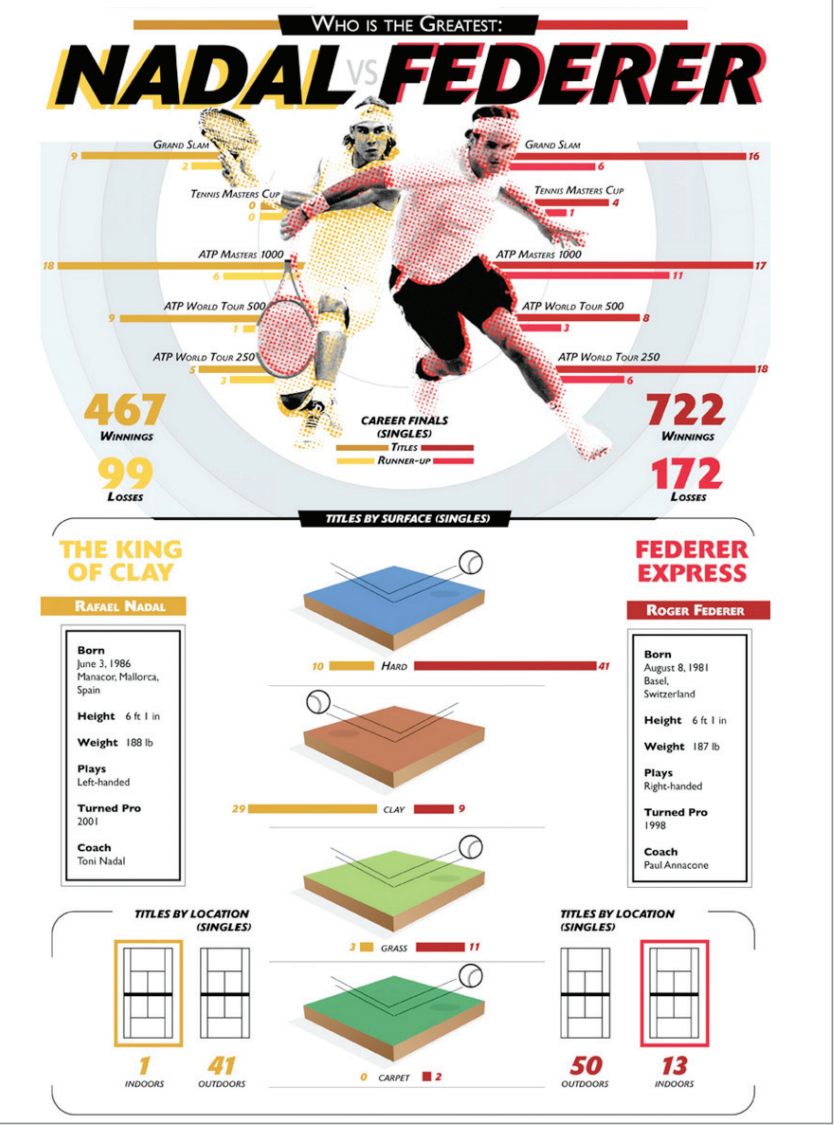

http://fosbury.cat/bloc/infografia-nadal-vs-federer-qui-es-el-millor

b) Deporte preferente en portada (sobre 65 censados)

-35 fútbol (33 fútbol profesional, 1 femenino, 1 sala)

-11 polideportivo

-7 baloncesto

-3 olimpismo

-2 economía y marketing deportivo

-2 ciclismo

-1 tenis

-1 deportes de EUA

-1 atletismo

-1 deporte adaptado

-1 hockey

c) Modelo de negocio (son mixtos, por eso suman más de 66)

-45 publicidad

-6 tienda, productos exclusivos o vintage

-3 venta de ejemplares en papel

-2 comunidad de lectores

-2 colaboración con otros medios

-1 donaciones

-2 asesoría

-1 periodismo de marca

-1 eventos y patrocinios

-7 desconocido, no identificado

Con estos datos principales, se ha realizado la comprobación inversa. Se ha identificado qué proyectos emplean o plantean modelos de negocio alternativos al convencional basado en publicidad o venta de ejemplares. Con esta segunda revisión, se encuentra la segunda confirmación de la hipótesis de trabajo. La ausencia de publicidad al uso requiere un modelo comercial diferente, que permite la publicidad pero abre la puerta a otros ingresos. Se analizan de forma cualitativa ocho casos concretos que validan la hipótesis:

\section{Elenganche.es \\ http://www.elenganche.es}

Ofrece Historias de fútbol, un género novedoso, de lectura pausada y muy elaborada. Cuenta con un diseño cuidado (fotografía, ilustración, adaptado a móviles y tabletas) y una extensa gama de detalles. Se aleja de la actualidad para conectar con lectores interesados en el fútbol profesional en un sentido más amplio. Utiliza géneros como el reportaje histórico o la viñeta. La propuesta de valor se centra en un lector que busca contenidos más allá de la lectura diaria. Se concreta en un modelo de negocio con dos vías de ingresos destacadas: la tienda y la suscripción. La primera vende productos textiles de moda, mientras que la segunda da acceso a una comunidad con contenido exclusivo. Apenas hay publicidad.

\section{Kaiser magazine football http://www.kaisermagazine.com}

Publica una revista en papel y digital sobre contenidos futbolísticos. No hay actualidad, sino reportajes en profundidad, historias del mundo del fútbol, guías que analizan equipos actuales e históricos y otros contenidos de fútbol clásico. La propuesta de valor es muy clara, con contenidos muy cuidados en la redacción, diseño y selección de temas. El modelo de negocio se basa en la venta de ejemplares premium de su revista bimestral, así como en la venta de láminas y otros contenidos exclusivos. Esta extensión de la marca convierte a Kaiser en un medio innovador, que pone en valor su propio diseño.

\section{Sorprende el escaso grado de especiali- zación y diversificación de los proyectos}

\section{Volata \\ http://volatamag.cc}

Se dedica al ciclismo profesional. Es la única revista de calidad en papel y en digital que se edita en España. Emplea distintos géneros: reportajes y entrevistas de formato largo, análisis de la situación del deporte nacional e internacional, así como otros artículos de opinión. No hay actualidad al uso. La innovación procede de la calidad de la producción, la apuesta por un nicho de mercado y la creación de una tienda con servicios y productos propios. Incluye publicidad y venta de ejemplares en papel.

\section{Líbero \\ http://shop.revistalibero.com}

Es una revista trimestral en papel que se vende por suscripción, aunque se distribuye de forma puntual en una red seleccionada de quioscos. El género preferente es el reportaje, que no está accesible en la web. Ésta actúa como repositorio de contenidos, pero no como ventana. Los temas 
abarcan la relación entre fútbol y cultura, pero no se vincula con la competición profesional (resultados, clasificaciones), sino con sus personajes o los grandes temas. Presume de contenidos de lectura pausada.

\section{Panenka}

http://www.panenka.org

Tiene un enfoque parecido a la anterior, en la medida que se presenta como una publicación cultural sobre fútbol. Edita reportajes, historias de fútbol, entrevistas fuera de la actualidad y otros géneros o formatos interpretativos (libros). Encaja con la tendencia de lectura pausada, no pendiente de la actualidad. La revista mensual en papel contiene publicidad, pero tiene otras vías de ingresos de acuerdo con su propuesta de valor. Ha creado el Club Belgrado'76 para socios y suscriptores, así como una aplicación muy competitiva en el entorno móvil.

\section{Fosbury}

http://fosbury.cat/\#!menu

Es de temática polideportiva y de forma significativa no incluye el fútbol profesional en su agenda. Mezcla reportajes y entrevistas con deportistas de primer nivel en su deporte, pero no se centra en la competición o la clasificación puntual. Utiliza nuevas narrativas muy interesantes como las infografías y los reportajes interactivos. Además innova en el soporte, que es específico para tabletas y en lengua catalana. Contiene publicidad, pero su propuesta de valor hace hincapié en la idea de comunidad (socios lectores) y tienda.

\section{Maratón Radio \\ http://www.maratonradio.com}

Su propuesta es innovadora en la medida que consigue crear un proyecto periodístico y orientarlo a un mercado en auge. Este enfoque le permite centrarse en el atletismo amateur, las carreras populares y el triatlón. Además, la producción es innovadora: utiliza medios audiovisuales (radio) para que se pueda descargar y escuchar mientras se practica deporte. Por eso no se centra en la actualidad. Los ingresos mezclan la publicidad con la organización de eventos y los patrocinios.

Prima la repetición o la traslación de modelos convencionales al entorno digital

\section{Martiperarnau.com \\ http://www.martiperarnau.com}

Su propuesta es en esencia el fútbol, aunque recoge otros deportes. Tiene un estilo propio muy definido, lo que le permite publicar artículos de opinión y reportajes interpretativos de la realidad deportiva. Procura no vincular sus textos únicamente a resultados semanales, porque su gama de productos y servicios periodísticos requiere más tiempo de producción. Publican y venden una revista semestral, informes sobre temas especiales y otras publicaciones de corte menor. Cuentan con publicidad, pero la propuesta de valor gira en torno al club de socios, que tienen acceso a contenidos especiales.

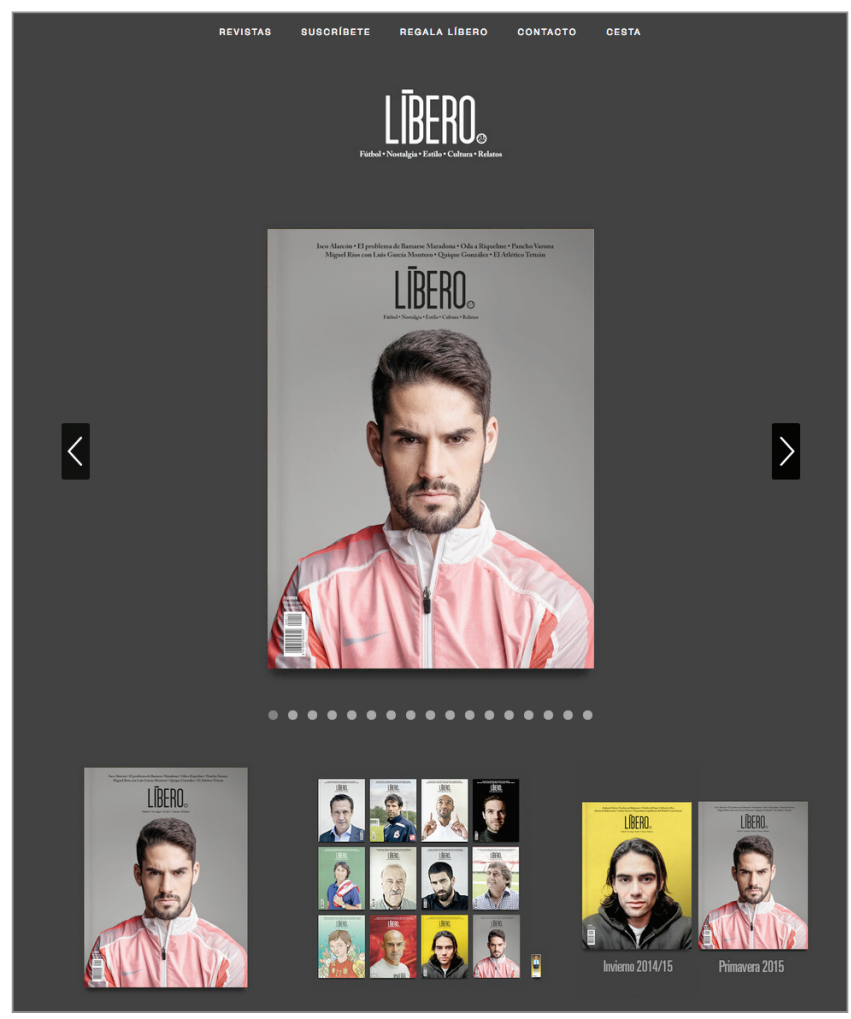

http://shop.revistalibero.com

Hay que señalar que se encontraron fórmulas innovadoras en alguno de los campos propios de actividad periodística. El periodismo deportivo ha sido propicio al patrocinio y a fórmulas publicitarias novedosas. Por eso, es innovadora la propuesta de Kiaenzona.com, una web de baloncesto que responde al ahora denominado periodismo de marca. Es un patrocinio de la liga profesional de baloncesto español, que incluye un rincón para aficionados, espacio para la interacción y una novedosa apuesta por el juego (gamification). Contiene videonoticias, reportajes y actualidad.

http://kiaenzona.com

La innovación de Eldesmarque.com procede del modelo de producción periodística. Es el único periódico que ha utilizado la estrategia de extensión de marca. El género es actualidad informativa y noticias, pero el modo en que se desarrolla sí es innovador. La extensión de marca permite la creación de nuevos medios adaptados al público local bajo el paraguas de una marca reconocida y estable en el mercado periodístico. Es el único caso que innova en la propuesta de valor, pero no a través del género periodístico, sino de la especialización local. Abre nuevos mercados con el empleo de una solución tecnológica periodística.

http://eldesmarque.com

Otra innovación es la creación de productos en el nicho al que se dirigen. Destacan dos publicaciones dedicadas en exclusiva al fútbol profesional inglés, una web de deportes profesionales practicados en Estados Unidos, una revista de fútbol femenino, dos publicaciones sobre economía y marketing deportivo, otra final sobre deporte adaptado a las personas con alguna discapacidad y otra al hockey. La innovación consiste en la atención a un mercado sin atender para el público español. 


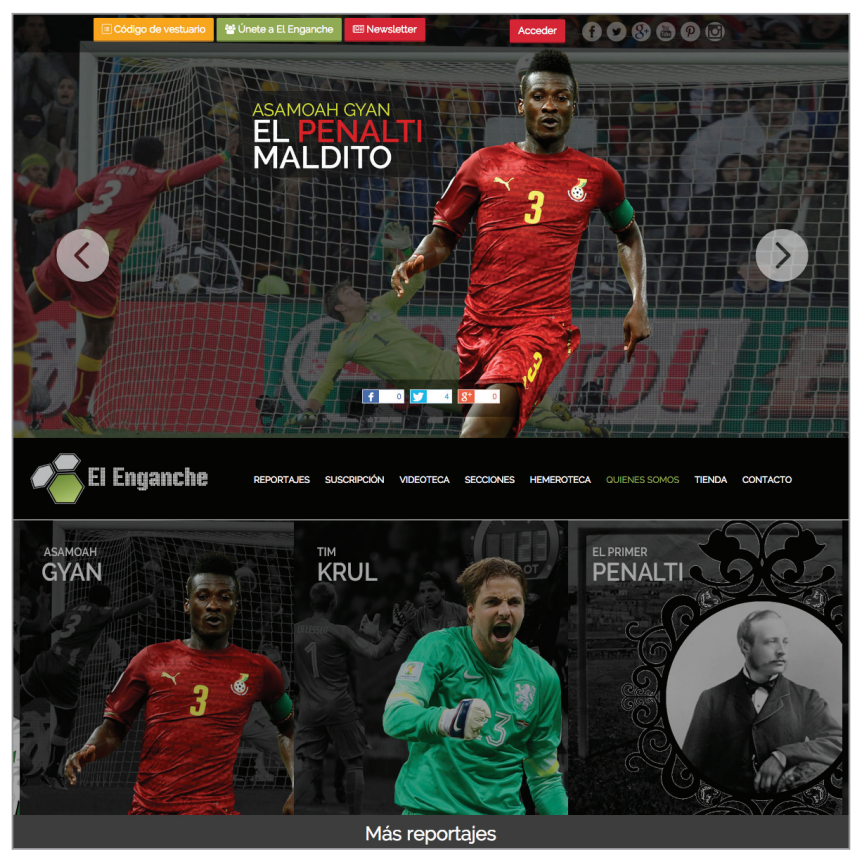

http://www.elenganche.es

En cuanto a las fórmulas de financiación, encontramos innovadora la oferta de profesionales de un deporte para el seguimiento de un club, una liga o una promesa. Metadeporte. com ofrece este tipo de servicio. La redacción profesional es un servicio de valor añadido que puede financiar proyectos periodísticos, como sucede en la prensa económica. http://metadeporte.com

También destaca la originalidad de los álbumes y cromos personalizados de EIDeporteConquense.com, que ha creado una serie de estampas de clubes locales de fútbol. http://eldeporteconquense.com

\section{Conclusiones}

La primera conclusión se centra en el producto: se observa poca innovación. Prima la repetición o la traslación de modelos convencionales al entorno digital. La multiplicación de webs deportivas dedicadas a la actualidad y las noticias del fútbol profesional revela poca innovación en la creación de mercados, augura poca sostenibilidad o viabilidad empresarial y limita la eficiencia del proyecto.

Destaca la escasa innovación audiovisual. Hay pocas iniciativas audiovisuales puras. El desarrollo de productos radiofónicos tiene un coste de producción mínimo, por lo que es una oportunidad interesante para diferenciarse. Sí merece la pena reseñar el empleo de música y multimedia por parte de Ecosdelbalon.com.

Se observa poca atención al diseño como parte del mensaje periodístico. La mayoría de las publicaciones emplean plantillas estandarizadas o poco elaboradas. Este look-andfeel debilita la primera visita al medio. La reputación de la propuesta innovadora es un elemento que contribuye al éxito del proyecto (Luoma-Aho; Nordfors, 2009). En el censo analizado, la gestión de la reputación no aparece como una prioridad. No hay una gestión intensiva de las redes sociales. No hay acciones concretas en este sentido, salvo seguimientos de partidos o comentarios.

La segunda conclusión trata de la propuesta de valor, en particular el modelo de negocio. La captación de grandes anunciantes y grandes masas de publicidad no cuadra con el periodismo emprendedor. Cuesta hacerse hueco y generar audiencia masiva para que los números de la inversión publicitaria cuadren con cpm (coste por mil), click-through o pay-per-click. Asimismo, la creación de un medio nuevo que compita con el dominio preferente de los convencionales tanto en prensa (Marca, As o el diario local) como en televisión parece una aventura difícil. La diferenciación por la vía del producto o el servicio tiende a cero. Además, el lector no paga por servicios no diferenciados, por lo que su rentabilidad tiende también a cero. Estos usuarios activos no se concretan en una inversión publicitaria masiva. Esto vacía los posibles desarrollos estratégicos y anula la monetización por la vía de la audiencia masiva. Con una inversión y recursos limitados, hay que ofrecer un servicio periodístico muy diferente, bien en el mercado (geográfico o especialidad deportiva) o en la narrativa (géneros no noticiosos).

El enfoque en productos y mercados saturados reduce las posibilidades de éxito de una iniciativa periodística. En una economía basada en la atención de la audiencia, la sobreabundancia de la oferta ahoga las nuevas propuestas. Los proyectos deportivos estudiados compiten con los medios convencionales, con las secciones deportivas de los medios generalistas y también con todo tipo de

http://volatamag.cc/revista 
periodistas y aficionados que publican contenidos en redes sociales. En una futura investigación, sería relevante conocer qué opinión tienen los directores, cómo ven la innovación periodística y qué resultados produce en la audiencia el lanzamiento de servicios periodísticos innovadores. La entrevista en profundidad o la etnografía de las redacciones parecen métodos adecuados.

En síntesis, la innovación es escasa en la mayoría de los nuevos medios periodísticos deportivos españoles. En cambio, los proyectos emprendedores que han apostado por una propuesta de valor diferenciada sí han conseguido crear modelos de financiación basados en el pago (por parte del lector) y otras fórmulas complementarias. Esta mezcla de innovación en el género periodístico y la propuesta de valor contribuye a crear un producto o servicio periodístico diferente, con alto valor añadido, y potencia el protagonismo del lector/consumidor que es capaz de diferenciar la calidad del producto.

\section{Notas}

1. El fordismo es un sistema de producción en serie. Promueve la especialización, la transformación del esquema industrial y la reducción de costos.

2. Esta investigación ha contado con el apoyo de Periodismo emprendedor, una esperanza renovada, contrato de I+D de la Universidad de Castilla-La Mancha con financiación del Departamento de Estado de Estados Unidos.

Inicio de la investigación: abril de 2014.

Conclusión de la investigación: enero de 2015.

\section{Bibliografía}

Alcoba-López, Antonio (2005). Periodismo deportivo. Madrid: Editorial Síntesis. ISBN: 9788497562737

Asociación de la Prensa de Madrid (2014). Informe anual de la profesión periodística. Madrid; APM, 112 pp.

http://www.apmadrid.es/publicaciones/informe-anual-dela-profesion-periodistica

Barrero-Muñoz, José (2008). Periodistas deportivos contra la violencia en el fútbol, al pie de la letra. Madrid: Fragua. ISBN: 9788470742552

Cabezuelo-Lorenzo, Francisco (2013). "Cinco años de crisis en el mercado de la comunicación (2008-2013)". Historia y comunicación social, v. 18, pp. 703-715.

http://dx.doi.org/10.5209/rev_HICS.2013.v18.44358

Casero-Ripollés, Andreu (2010). "Prensa en internet: nuevos modelos de negocio en el escenario de la convergencia". El profesional de la información, v. 19, n. 6, pp. 595-600.

http://www.elprofesionaldelainformacion.com/contenidos/2010/ noviembre/05.html

http://dx.doi.org/10.5209/10.3145/epi.2010.nov05

Casero-Ripollés, Andreu; Cullell-March, Cristina (2013). "Periodismo emprendedor. Estrategias para incentivar el autoempleo periodístico como modelo de negocio". Estudios sobre el mensaje periodístico, v. 19, pp. 681-690.

http://dx.doi.org/10.5209/rev_ESMP.2013.v19.42151

Chesbrough, Henry (2010). "Business model innovation:
Opportunities and barriers". Long range planning, abril-junio, v. 43, n. 2-3, pp. 354-363.

http://dx.doi.org/10.1016/j.Irp.2009.07.010

Goyanes, Manuel; Peinado, Fernando (2014). “Online newspapers business models in Spanish science journals: a review and suggestions for future research". Ámbitos. Revista internacional de comunicación, v. 24, pp. 91-100.

http://goo.gl/uS850x

Hedman, Jonas; Kalling, Thomas (2003). "The business model concept: Theoretical underpinnings and empirical illustrations". European journal of information systems, n. 12, pp. 49-59

http://dx.doi.org/10.1057/palgrave.ejis.3000446

Infoadex (2014). Estudio InfoAdex de la inversión publicitaria en España 2014. Madrid: Infoadex.

http://www.infoadex.es/resumen_estudio_2014.pdf

Luoma-Aho, Vilma; Nordfors, David (2009). "Attention and reputation in the innovation economy". Innovation journalism, v. 6, n. 2.

http://www.innovationjournalism.org/archive/injo-6-2.pdf

Manfredi-Sánchez, Juan-Luis (coord.) (2015). Innovación y periodismo. Emprender en la universidad. Tenerife: Cuadernos artesanos de comunicación, n. 76.

http://www.cuadernosartesanos.org/2015/cac76.pdf

Manfredi-Sánchez, Juan-Luis; Artero, Juan-Pablo (2014). "New business models for the media: the Spanish case". En: Psychogiopoulou, Evangelia (ed.). Media policies revisited. The challenge for media freedom and independence. Palgrave Macmillan, pp. 160-174. ISBN: 9781137337832

Manfredi-Sánchez, Juan-Luis; Rojas-Torrijos, José-Luis; Herranz-de-la-Casa, José-María (2015). "Periodismo emprendedor: el periodismo deportivo en España". Revista latina de comunicación, n. 70, pp 69-90 http://www.revistalatinacs.org/070/paper/1035-UC/05es.html http://dx.doi.org/10.4185/RLCS-2015-1035

Paniagua-Rojano, Francisco-Javier; Gómez-Aguilar, Marisol; González-Cortés, María-Eugenia (2014). "Incentivar el emprendimiento periodístico desde la Universidad". Revista latina de comunicación social, n. 69, pp. 548-570.

http://dx.doi.org/10.4185/RLCS-2014-1024

Rojas-Torrijos, José-Luis (2011). Periodismo deportivo de calidad. Madrid: Fragua. ISBN: 9788470744594

Rojas-Torrijos, José-Luis (2013). "Periodismo deportivo. Hacia la innovación y el emprendimiento en la Red". En: Sobrados-León, Maritza (coord.). Presente y futuro en el periodismo especializado. Madrid: Fragua, pp. 197-247. ISBN: 9788470745553

Universidad Miguel Hernández (2014). Ranking de innovación periodística 2014. Elche: Grupo de Investigación de la Comunicación.

http://mip.umh.es/ranking

Yuste, Bárbara; Cabrera, Margarita (2014). Emprender en periodismo. Nuevas oportunidades para el profesional de la información. Barcelona: Editorial UOC. ISBN: 978 8490640708 